\title{
A Diet with 3\% of Energy from a Mixture of Omega-3 Fatty Acids Significantly Increases in Vivo Lipid Peroxidation in Postmenopausal Women
}

\author{
A. Saari Csallany ${ }^{*}$, Cheryl E. Ainslie-Waldman ${ }^{1,2}$, Lindsay R. Young1, Chi Chen1, Mindy S. Kurzer ${ }^{1}$, \\ Susan K. Raatz ${ }^{1,3}$
}

${ }^{1}$ Department of Food Science and Nutrition, University of Minnesota, Saint Paul, MN, USA

${ }^{2}$ Division of Epidemiology and Community Health, University of Minnesota, Minneapolis, MN, USA

${ }^{3}$ USDA Grand Forks Human Nutrition Center, Grand Forks, ND, USA

Email: *ascsalla@umn.edu

How to cite this paper: Csallany, A.S., Ainslie-Waldman, C.E., Young, L.R., Chen, C., Kurzer, M.S. and Raatz, S.K. (2016) A Diet with 3\% of Energy from a Mixture of Omega-3 Fatty Acids Significantly Increases in Vivo Lipid Peroxidation in Postmenopausal Women. Food and Nutrition Sciences, 7, 1099-1111.

http://dx.doi.org/10.4236/fns.2016.712105

Received: September 2, 2016

Accepted: October 16, 2016

Published: October 20, 2016

Copyright $\odot 2016$ by authors and Scientific Research Publishing Inc. This work is licensed under the Creative Commons Attribution International License (CC BY 4.0).

http://creativecommons.org/licenses/by/4.0/ (c) (i) Open Access

\begin{abstract}
Dietary omega-3 ( $\mathrm{n}-3)$ polyunsaturated fatty acids (PUFA) are recommended by public health organizations to reduce the risk of cardiovascular disease, and several epidemiological studies have suggested there is an inverse association between $n-3$ intake and human cancers. However, $\mathrm{n}-3$ are susceptible to an increase in lipid peroxidation in the human body. As part of a crossover dietary intervention study of a diet (20\% of energy from fat) with or without an additional $3 \%$ of energy from a mixture of $\mathrm{n}-3$ (with $5.36 \mathrm{~g} \alpha$-linolenic acid and $1.45 \mathrm{~g}$ eicosapentaenoic acid and docosahexaenoic acid per $2000 \mathrm{kcal}$ per day), we measured total in vivo lipid peroxidation in healthy postmenopausal women $(n=15)$. Our results indicated that the diet with $3 \%$ of energy from $n-3$ significantly increased the urinary concentrations of total polar lipophilic aldehydes and related compounds produced via lipid peroxidation $(p<0.05)$ as well as the $\alpha, \beta$-unsaturated hydroxy aldehydes 4 -hydroxy-2-transhexenal $(p<0.05)$ and 4 -hydroxy-2-trans-decenal $(p<0.05)$ compared to the diet with less than $1 \%$ of energy from $n-3$. This is also the first study to document the presence of 4-hydroxy-2-trans-decenal in the urine of individuals consuming $n-3$. These results demonstrate that an increase in $3 \%$ of energy from dietary $n-3$ increases in vivo lipid peroxidation.
\end{abstract}

\section{Keywords}

Lipid Peroxidation, $\mathrm{n}-3$ Fatty Acids, Human, HHE, 4-Hydroxyhexenal, HDE, 4-Hydroxydecenal, $\alpha, \beta$-4-Hydroxyaldehydes, HPLC, MS 


\section{Introduction}

Lipid peroxidation is an autocatalytic, free radical-induced process by which polyunsaturated fatty acids (PUFA) are degraded into lipid hydroperoxides and reactive lipophilic aldehydes and ketones. This process can occur in food products as well as in biological membranes, where the production of lipophilic $\alpha, \beta$-unsaturated hydroxyaldehydes such as 4-hydroxy-2-trans-hexenal (HHE) and 4-hydroxy-2-trans-nonenal (HNE) may result in cellular damage or death [1] [2]. The work of Esterbauer and colleagues established that such 4-hydroxyalkenals were highly reactive and could bind with most amino acids, and that HNE had been implicated in the etiology of atherosclerosis, diabetes, and neurodegenerative diseases [2]-[4]. In addition to vitamin E deficiency and impaired endogenous antioxidant systems, a diet high in PUFA would be expected to increase total lipid peroxidation within an organism [5]-[9]. Previous research from our laboratory demonstrated that heating methyl linolenate, formed from the omega-3 ( $\mathrm{n}-$ 3) PUFA $\alpha$-linolenic acid (ALA, 18:3 n - 3), yielded detectable quantities of HHE [10], but limited human research had documented its production via in vivo lipid peroxidation following $\mathrm{n}-3$ consumption [11].

As a secondary analysis of an NIH-funded, dietary crossover study evaluating $\mathrm{n}-3$ intake and breast cancer risk [12], our objective was to evaluate in vivo lipid peroxidation in response to a diet with or without foods rich in $n-3$ (e.g., salmon, tuna, flaxseed oil, and walnuts). Briefly, each study participant $(\mathrm{n}=15)$ consumed meals providing $3 \%$ or less $1 \%$ of energy from $n-3$ for eight weeks; energy needs were computed to maintain body weight based on the Harris-Benedict equation. After a washout period of six to eight weeks, each study participant completed the other diet. Diets were similar with respect to macronutrient distribution, except for the differences in $n-3$. The diet with $3 \%$ of energy from $n-3$ provided approximately 5.36 g ALA and 1.45 g eicosapentaenoic acid (EPA, 20:5 n-3) and docosahexaenoic acid (DHA, 22:6 n - 3) from food sources per $2000 \mathrm{kcal}$ per day [12].

Previously, this laboratory developed a method based on the work of Esterbauer [13] to identify and quantify the urinary excretion of polar and nonpolar aldehydic products of in vivo lipid peroxidation in animal models and validated this method in humans [5] [14]-[16]. Increased in vivo lipid peroxidation was measured in rats fed a standard diet compared to fasted rats, suggesting that the formation of these compounds was at least partially influenced by diet [14]. Further research demonstrated that rats were subjected to a vitamin E deficiency, consuming a diet high in PUFA (including cod liver oil), or administered that carbon tetrachloride exhibited increased urinary excretion of lipophilic aldehydes and related compounds [15]. Administration of vitamin E or probucol significantly reduced the increased in vivo lipid peroxidation observed in a ratmodel of diabetes, indicating that antioxidants inhibited the formation of several reactive aldehydes [5]. However, no research has been conducted on the response of in vivo lipid peroxidation to a well-controlled human feeding study including a mixture of ALA, EPA, and DHA. We hypothesized that the diet with $3 \%$ of energy from $n-3$ would significantly increase in vivo lipid peroxidation in general and the excretion of HHE in particular. 


\section{Material and Methods}

\subsection{Chemicals and Supplies}

2,4-Dinitrophenylhydrazine (DNPH), hexanal (98\%), pentan-2-one (97\%), hept-2-enal (97\%), hepta-2,4-dienal (90\%), decanal, deca-2,4-dienal, methanol, and water were purchased from Sigma-Aldrich (St. Louis, MO). Hydrochloric acid and methylene chloride were obtained from J.T. Baker (Philipsburg, NJ). Acetone was purchased from Fisher Scientific (Fair Lawn, NJ), and hexane was purchased from EMD Chemicals (Gibbstown, NJ); butanone, octanal, non-2-enal, and DNPH-derivative of butyraldehyde from Supelco Analytical (Bellefonte, PA). DNPH-derivatives of 4-hydroxy-2trans-octenal (HOE) and 4-hydroxy-2-trans-decenal (HDE) were generously provided previously by Dr. Esterbauer's laboratory, University of Graz (Graz, Austria). HHE and HNE were purchased from Cayman Chemicals (Ann Arbor, MI). $L$-ascorbic acid was procured from Eastman (Rochester, NY). All chemicals and solvents were high-performance liquid chromatography (HPLC)-grade and filtered and degassed prior to use.

\subsection{Instrumentation}

The HPLC system included a Varian 9010 solvent delivery system (Varian, Walnut Creek, CA), a Waters WISP 710B sample injector (Waters, Milford, MA), an Ultrasphere ODS C18 reversed-phase column $(25 \mathrm{~cm} \times 4.6 \mathrm{~mm}$ i.d., $5 \mu \mathrm{m}$ particle size) (Beckman, Fullerton, CA) equipped with a $7.5 \times 4.6 \mathrm{~mm}$ guard column (Alltech Associates, Deerfield, IL), and a Varian 9050 variable wavelength UV-VIS detector (Varian, Walnut Creek, CA). The integration of peaks was completed with Varian Star Chromatography Workstation (Varian, Walnut Creek, CA) software installed on a computer connected to the detector.

\subsection{Study Population and Diets}

Complete inclusion and exclusion criteria have been provided by Young et al. [12]. Healthy postmenopausal women with a body mass index between 19 and $32 \mathrm{~kg} / \mathrm{m}^{2}$ were eligible to participate in the original study. The subjects $(n=15)$ were randomly assigned to an isoenergetic diet with $20 \%$ of energy from fat with $(+n-3)$ or without $(-n-3) 3 \%$ of energy from $n-3$ daily for eight weeks and subsequently consumed the other study diet for the same period of time following a six- to eight-week washout period. The $+\mathrm{n}-3$ diet macronutrient composition (\% energy) per $2000 \mathrm{kcal}$ was as follows: $317 \mathrm{~g}$ carbohydrate (62\%), $77 \mathrm{~g}$ protein (15\%), $54 \mathrm{~g}$ fat (23\%), $25.2 \mathrm{~g}$ fiber, $210 \mathrm{mg}$ cholesterol, $14.8 \mathrm{~g}$ saturated fatty acids (SFA), $16.2 \mathrm{~g}$ monounsaturated fatty acids (MUFA), 16.1 g PUFA, $6.82 \mathrm{~g} \mathrm{n}-3$, $5.36 \mathrm{~g}$ ALA, $0.46 \mathrm{~g}$ EPA, and $0.99 \mathrm{~g}$ DHA. The $-\mathrm{n}$ - 3 diet macronutrient composition (\% energy) per $2000 \mathrm{kcal}$ was as follows: $329 \mathrm{~g}$ carbohydrate (65\%), $76 \mathrm{~g}$ protein (15\%), $46 \mathrm{~g}$ fat (20\%), $23.9 \mathrm{~g}$ fiber, $203 \mathrm{mg}$ cholesterol, 13.4 g SFA, 13.9 g MUFA, 13.3 g PUFA, $0.88 \mathrm{~g} \mathrm{n}-3$, $0.85 \mathrm{~g}$ ALA, $0 \mathrm{~g}$ EPA, and $0.03 \mathrm{~g}$ DHA [12]. Baseline dietary intake was collected via food frequency questionnaire for 14 of the 15 subjects. Plasma phospholipid $n-3$ concentrations were significantly higher 
when subjects followed the $+n-3$ diet compared to the $-n-3$ diet [12]. The study was approved by the US Army Medical Research and Material Command's Human Subjects Research Review Board and the University of Minnesota Committee for the Use of Human Subjects in Research (study \#0307M49927), and the study was conducted in accordance with The Code of Ethics of the World Medical Association (Declaration of Helskini).

\subsection{Twenty-Four Hours Urine Collection Protocol}

As part of the original study, each subject provided two consecutive 24-hour urine collections during the final week of each diet [12]. The samples were kept in an opaque, $3.5 \mathrm{~L}$ jug with $1 \mathrm{~g}$ ascorbic acid at refrigerator temperature throughout the collection. The collections were pooled, and aliquots were stored at $-80^{\circ} \mathrm{C}$ prior to analyses. Urinary creatinine was measured for each sample by rate reflectance spectrophotometry using an Ortho Clinical Diagnostics Vitros analyzer at the University of Minnesota Medical Center, Fairview, Diagnostic Laboratories.

\subsection{Preparation of DNPH Reagent and DNP-Hydrazone Standards}

The DNPH reagent was prepared daily following the method in Kim et al. [14]. $12.5 \mathrm{mg}$ of DNPH, recrystallized three times, was mixed the $25 \mathrm{~mL}$ of $1 \mathrm{~N}$ hydrochloric acid at $50^{\circ} \mathrm{C}$ for approximately one hour. Following cooling, the reagent was rinsed four times with hexane to remove any impurities. DNP-hydrazone standards were prepared from pure standards (e.g., hexanal, HHE) following the method of Kim et al. [14].

\subsection{Formation and Isolation of DNP-Hydrazones of Urinary Aldehydes and Related Compounds}

Three $\mathrm{mL}$ of urine samples in duplicate were reacted with an equal volume of DNPH reagent overnight with agitation at room temperature. A reagent blank and acetoneDNPH standard were prepared by reacting an equal amount of DNPH reagent or $1 \%$ acetone/water ( $\mathrm{v} / \mathrm{v})$, respectively, and DNPH reagent. The following day, the reaction mixtures were extracted three times with $10 \mathrm{~mL}$ methylene chloride, and the organic phases were separated via centrifugation for 10 minutes at $1360 \times g$. The sample extracts were pooled and evaporated to $500 \mu \mathrm{L}$ under $\mathrm{N}_{2}$ gas. Each sample was applied to two thin-layer chromatography (TLC) plates, and the plates were developed with methylene chloride at room temperature for approximately one hour for primary separation.

Nonpolar and polar compounds were isolated by comparison to the $\mathrm{R}_{\mathrm{f}}$ values of acetone-DNPH (0.55) and DNPH reagent blank (0.23). The polar carbonyl compounds were found between the origin and $\mathrm{R}_{\mathrm{f}} 0.23$, while the nonpolar carbonyl compounds were found between $R_{f} 0.55$ and the solvent front. Osazones were isolated between the acetone-DNPH and DNPH reference bands and were discarded from the following analyses. The polar and nonpolar carbonyls compounds from the TLC plates were cut and eluted three times with $10 \mathrm{~mL} 100 \%$ methanol. The pooled extracts were evaporated to approximately $5 \mathrm{~mL}$ under $\mathrm{N}_{2}$ gas and centrifuged for 20 minutes at $1360 \times g$ to 
remove any silica. The clear supernatants were evaporated to less than $1 \mathrm{~mL}$ and made up to exactly $1 \mathrm{~mL}$ with $100 \%$ methanol.

\subsection{Identification of DNP-Hydrazones}

Aliquots of the polar and nonpolar DNP-hydrazones were analyzed separately via HPLC with two different solvent systems [10] [14]. Prior to injection into the HPLC system, the methanol extracts were filtered through a $0.45 \mu \mathrm{m}$ filter. Aliquots of polar carbonyls $(100 \mu \mathrm{L})$ were injected into the HPLC system, using isocratic elution for 10 minutes with $55 \%$ methanol $/ 45 \%$ water $(\mathrm{v} / \mathrm{v})$, followed by a linear gradient of $55 \%$ methanol/45\% water (v/v) for 20 minutes and 100\% methanol (v/v) for 10 minutes. The analysis of nonpolar carbonyls was identical, but the initial isocratic gradient was $75 \%$ methanol/25\% water (v/v). The absorbance of polar and nonpolar aldehydesand related carbonyl compounds was monitored at $378 \mathrm{~nm}$, and the rejection of peaks was set to 2000 area counts. The detection limit of the system was 1 nghexanal-DNPH per $50 \mu \mathrm{L}$ injection. Peaks were identified by comparison of retention times to known standards. Each sample was injected at least twice, and paired samples were run in the same series, whenever possible.

\subsection{Liquid Chromatography-Mass Spectrometry (LC/MS) Analysis of HDE-DNPH Adduct}

For representative subjects' following the $+n-3$ diet $(n=8)$, DNP-hydrazones were pooled in duplicate, and two samples were collected between 32 and 35 minutes of the polar HPLC method described above. LC/MS analysis was completed by following a previously published method [17]. A $5 \mu \mathrm{L}$ aliquot of the pooled samples was injected into a Waters Acquity ultra-performance liquid chromatography system (Milford, MA) and separated by a gradient of mobile phase ranging from water to $95 \%$ aqueous acetonitrile containing $0.1 \%$ formic acid over a ten minute run. LC eluate was introduced into a Waters SYNAPT QTOF mass spectrometer (Milford, MA) for accurate mass measurement and tandem MS (MS/MS) analysis. Capillary voltage and cone voltage for electrospray ionization was maintained at $-3 \mathrm{kV}$ and $-35 \mathrm{~V}$ for negative mode detection, respectively. Source temperature and desolvation temperature were set at 120 and $350^{\circ} \mathrm{C}$, respectively. Nitrogen was used as both cone gas $(50 \mathrm{~L} / \mathrm{h})$ and desolvation gas $(600 \mathrm{~L} / \mathrm{h})$ and argon as collision gas. For accurate mass measurement, the mass spectrometer was calibrated with sodium formate solution (range $\mathrm{m} / z 50-1000$ ) and monitored by the intermittent injection of the lock mass leucineenkephalin $\left([\mathrm{M}-\mathrm{H}]^{-}=\right.$ $554.2615 \mathrm{~m} / \mathrm{z}$ ) in real time. Mass chromatograms and mass spectral data were acquired and processed by MassLynx software (Waters) in centroid format. The presence of HDE-DNPH in the samples was confirmed by a comparison with the pure standard and MS/MS fragmentation.

\subsection{Statistical Analysis}

Data were log-transformed and analyzed in SAS v9.2 (SAS Institute Inc., Cary, NC) via 
paired Student's t-test. The cutoff for significance was set at $p=0.05$.

\section{Results}

\subsection{Subject Characteristics}

A summary of baseline characteristics for the subjects is shown in Table 1. The average age of the subjects was $56.21 \pm 5.72$ years old, and the average BMI was $27.70 \pm 3.38$ $\mathrm{kg} / \mathrm{m}^{2}$. Of the $3 \%$ of energy consumed from $\mathrm{n}-3$ fatty acids during the low-fat $+\mathrm{n}-3$ diet, approximately $80 \%$ was comprised of ALA with the remainder from EPA (6.9\%) and DHA (14.9\%) [12].

\subsection{Total Polar and Nonpolar DNP-Hydrazones}

Figure 1 shows the total area under the individually measured peaks from the polar and nonpolar HPLC systems. Following the $+n-3$ diet, urinary total polar DNP-hydrazones were significantly increased more than two-fold compared to the diet with less than $1 \%$ of energy from $n-3(p<0.05)$. In contrast, there was a modest increase in urinary nonpolar DNP-hydrazonesin the $+n-3$ compared to the $-n-3$ diet, but the difference did not reach statistical significance $(p=0.78)$.

Table 1. Baseline demographic and dietary characteristics of women participating in $n-3$ crossover feeding study.

\begin{tabular}{|c|c|}
\hline Baseline characteristic $(n=15)$ & Mean \pm SD \\
\hline \multicolumn{2}{|l|}{ Race/ethnicity, n (\%) } \\
\hline African-American & $1(6.67 \%)$ \\
\hline Hispanic-White & $1(6.67 \%)$ \\
\hline White & $13(86.67 \%)$ \\
\hline Age, y & $56.21 \pm 5.72$ \\
\hline Body mass index, $\mathrm{kg} / \mathrm{m}^{2}$ & $27.70 \pm 3.38$ \\
\hline Baseline diet $(\mathrm{n}=14)$ & Mean \pm SD \\
\hline Total energy, kcal & $1785 \pm 668$ \\
\hline Total fat, $g$ (\% energy) & $66.06 \pm 26.18(33.41 \% \pm 4.16 \%)$ \\
\hline Saturated fat, $g$ (\% energy) & $22.10 \pm 9.34(11.18 \% \pm 2.24 \%)$ \\
\hline Monounsaturated fat, $\mathrm{g}$ (\% energy) & $24.65 \pm 10.16(12.43 \% \pm 1.85 \%)$ \\
\hline Polyunsaturated fat, g (\% energy) & $14.35 \pm 10.16(7.28 \% \pm 0.69 \%)$ \\
\hline $18: 2 n-6, g$ & $12.87 \pm 4.69$ \\
\hline $18: 3 n-3, g$ & $1.19 \pm 0.46$ \\
\hline $20: 4 n-6, g$ & $0.086 \pm 0.048$ \\
\hline $20: 5 n-3, g$ & $0.021 \pm 0.037$ \\
\hline $22: 6 n-3, g$ & $0.055 \pm 0.037$ \\
\hline
\end{tabular}




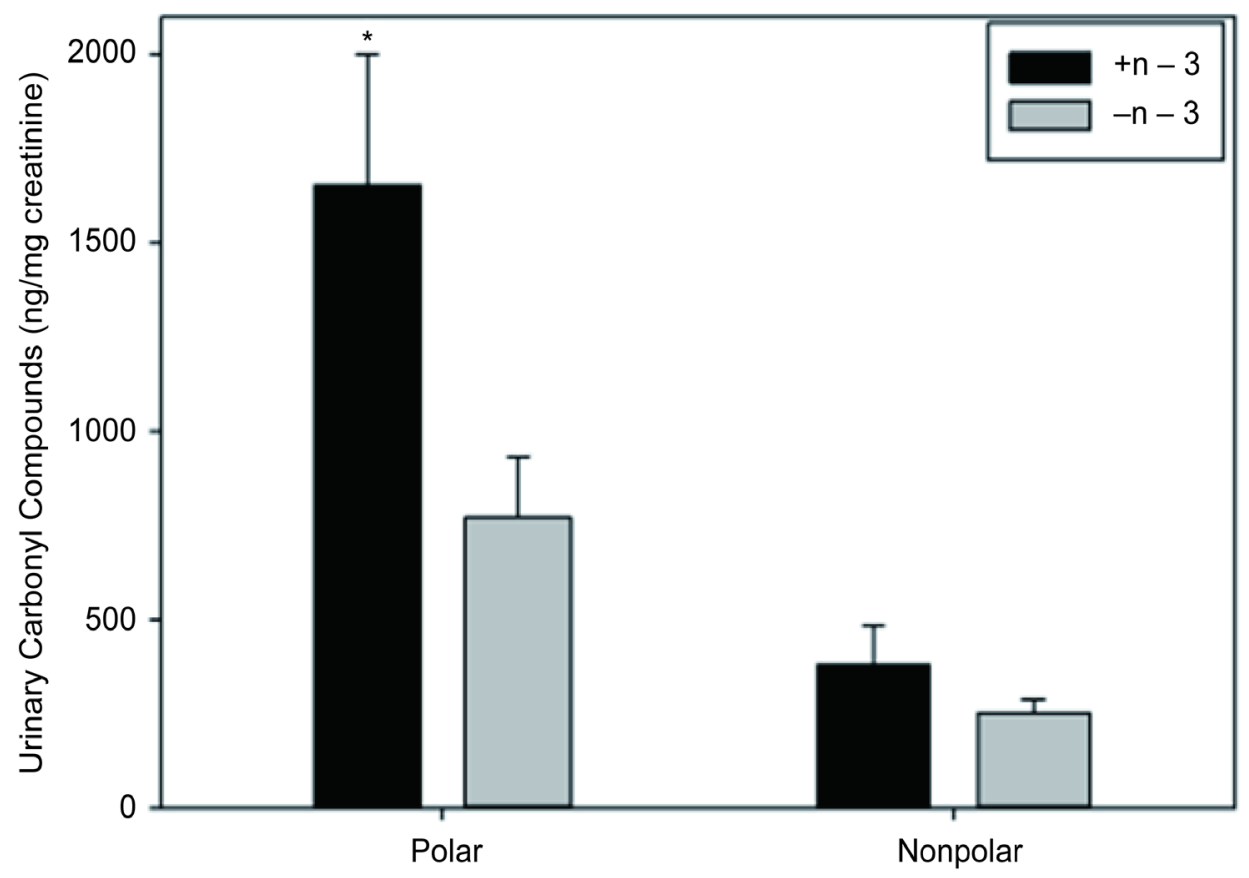

Figure 1. Total urinary polar and nonpolar lipophilic aldehydes following each 8 -week diet. ${ }^{\star} p<$ 0.05 .

\subsection{Individual Polar and Nonpolar DNP-Hydrazones}

A representative chromatogram of polar aldehydes is shown in Figure 2. The mean values for individual polar and nonpolar DNP-hydrazones are shown in Figure 3 and Figure 4, respectively. The urinary concentrations of HHE, HDE, and unidentified compounds $\mathrm{E}$ and $\mathrm{F}$ were significantly higher following the $+\mathrm{n}-3$ diet compared to the $-\mathrm{n}-3$ diet $(p<0.05)$. The difference in HNE concentrations did not reach statistical significance $(p=0.44)$. Although all nonpolar compounds quantified in the urine were slightly increased in the $+n-3$ versus $-n-3$ diet, none of the differences in concentrations reached statistical significance, likely due to the small number of subjects $(\mathrm{n}=15)$ and overall low concentrations of urinary nonpolar aldehydes. Figure 5 shows LC/MS traces confirming the presence of $\mathrm{HDE}$ in the urine samples of women consuming $n-3$.

\section{Discussion}

The present experiment is the first to measure a significant increase in total polar aldehydes and two of the $\alpha, \beta$-unsaturated hydroxy aldehydes HHE and HDE in the urine of individuals following a low-fat diet with $3 \%$ of energy from $n-3$. These results confirm our hypothesis that an increase in dietary $n-3$ increases in vivo lipid peroxidation. The highly unsaturated $\mathrm{n}-3$ are susceptible to lipid peroxidation and the formation of selected secondary lipid peroxidation productions including polar aldehydes, as shown in previous work by this laboratory [10] [15].

The present study is the first to quantify total urinary lipophilic polar and nonpolar 


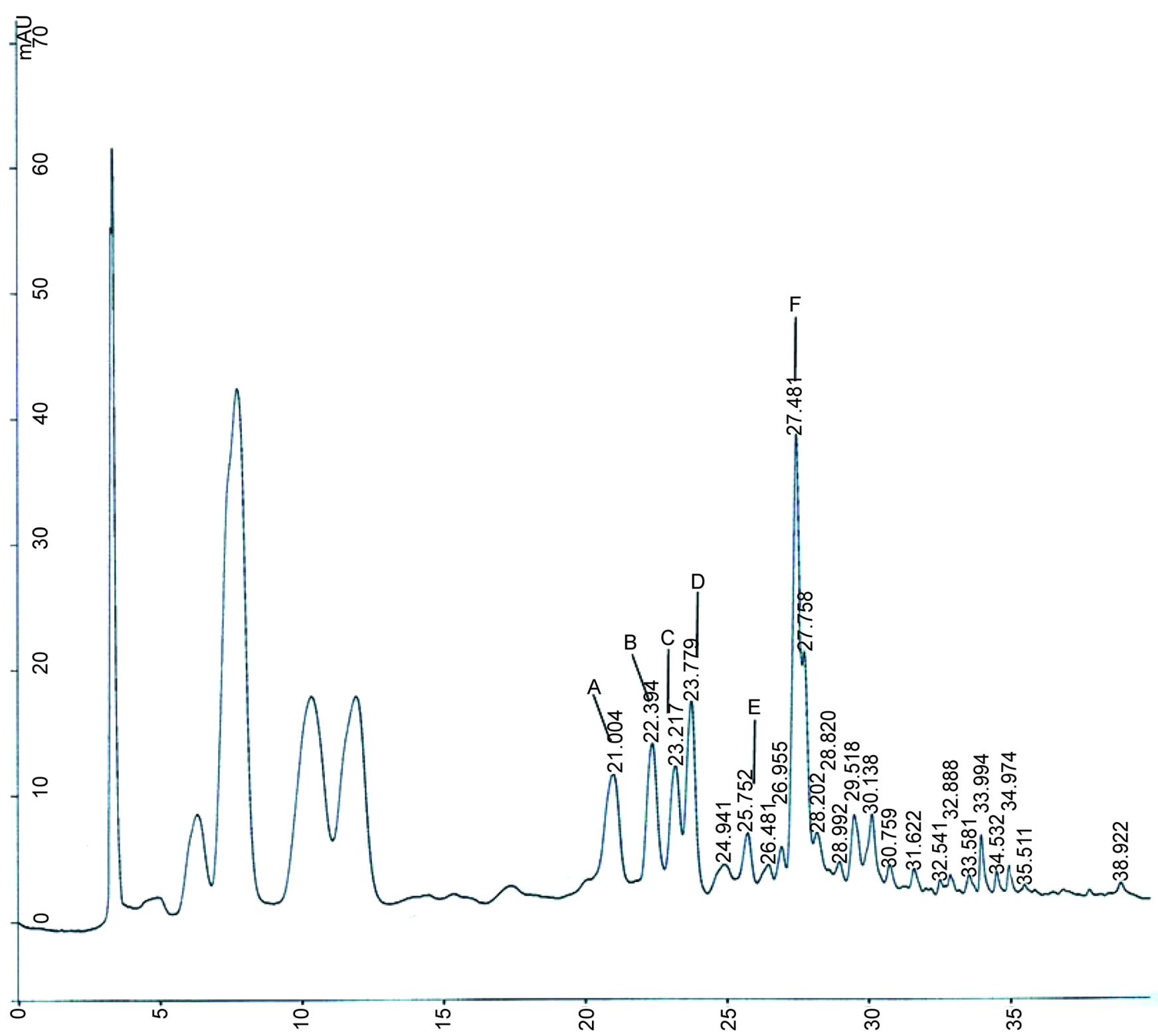

Figure 2. Representative HPLC chromatogram showing polar 2,4-dinitrophenylhydrazonesisolated from human urine. A = 4-hydroxy2-trans-hexenal (HHE), B = 4-hydroxy-2-trans-octenal (HOE), C = 4-hydroxy-2-trans-nonenal (HNE), D = 4-hydroxy-2-trans-decenal (HDE), $\mathrm{E}=$ unidentified compound, $\mathrm{F}=$ unidentified compound.

aldehydes and related carbonyl compounds from the in vivo lipid peroxidation of $\mathrm{n}-3$ in humans. This is also the first to report the presence of HDE from human urine (Figure 5). We documented significant increases in HHE, HDE, and two unidentified lipophilic polar carbonyl compounds in women consuming 3\% of energy from $n-3$ for eight weeks. This increase in HHE was expected due to its formation from lipid peroxidation of $\mathrm{n}-3$ [10] and confirmed the results of Calzada et al. [11] showing a significant increase in plasma HHE following DHA supplementation at 800 or $1600 \mathrm{mg}$ per day in men. Similar to Turley et al. [18], we did not detect a significant increase of $\mathrm{HNE}$ in individuals consuming the $+\mathrm{n}-3$ diet. This is not surprising given that thermal 


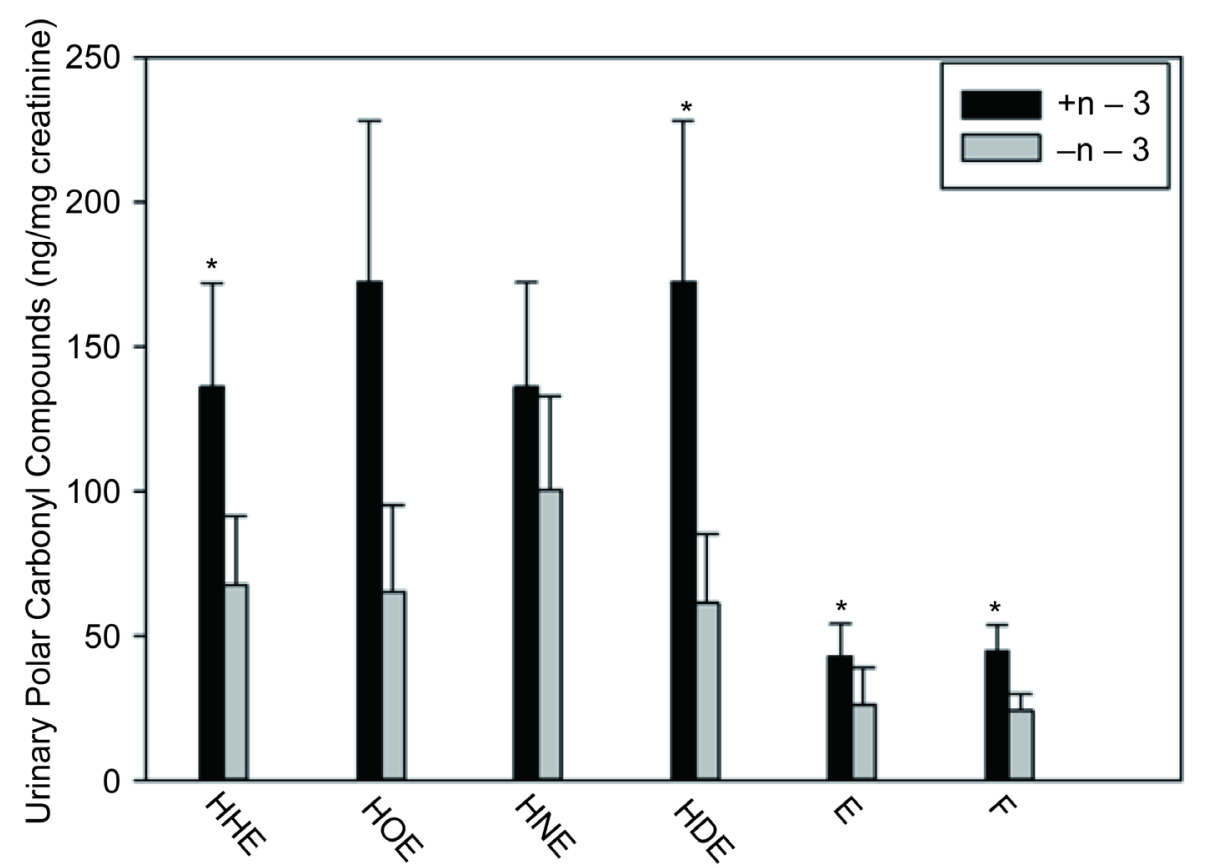

Figure 3. Individual polar urinary carbonyl compounds following each 8-week diet. HHE = 4-hydroxy-2-trans-hexenal, HOE = 4-hydroxy-2-trans-octenal, HNE = 4-hydroxy-2-trans-nonenal, $\mathrm{HDE}=4$-hydroxy-2-trans-decenal, $\mathrm{E}=$ unidentified compound, $\mathrm{F}=$ unidentified compound. ${ }^{\star} p<0.05$.

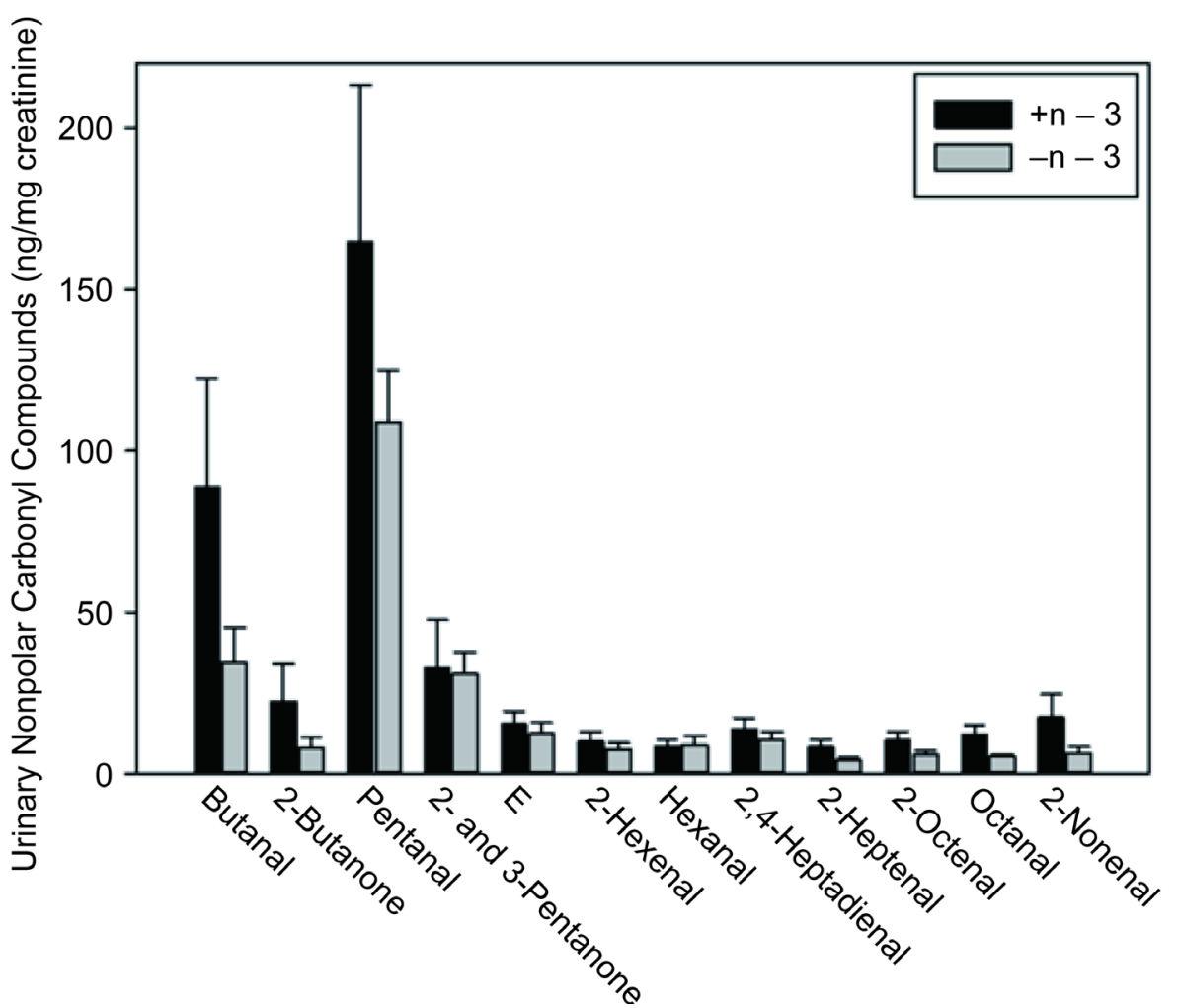

Figure 4. Individual nonpolar urinary compounds following each 8-week diet. $\mathrm{E}=$ unidentified compound. 
(a)

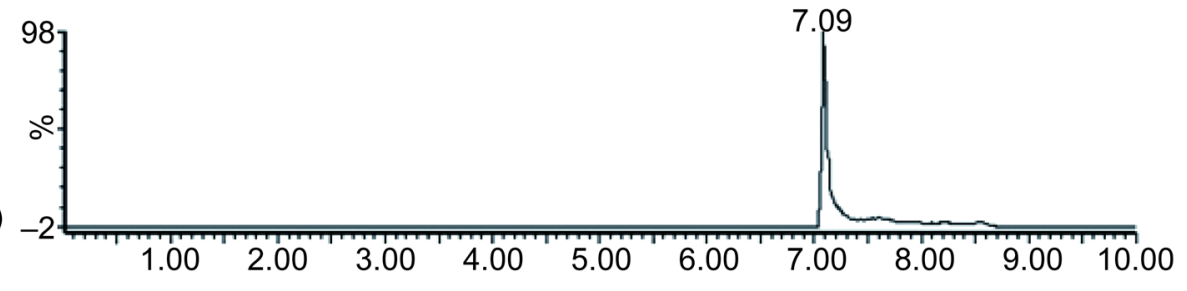

(b)

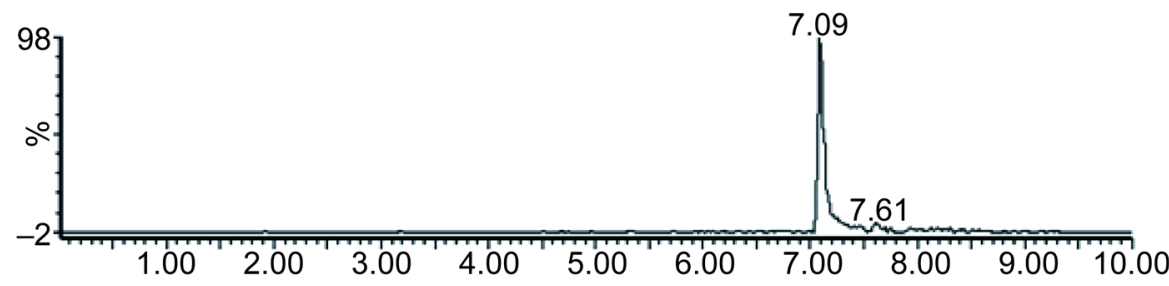

(c)

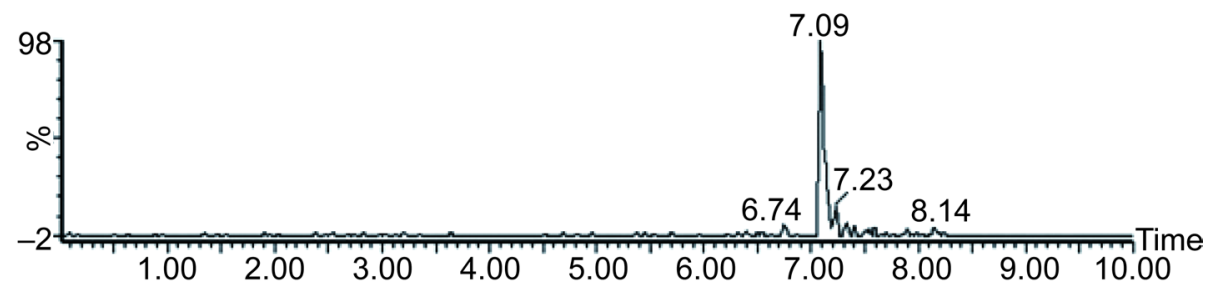

(d)

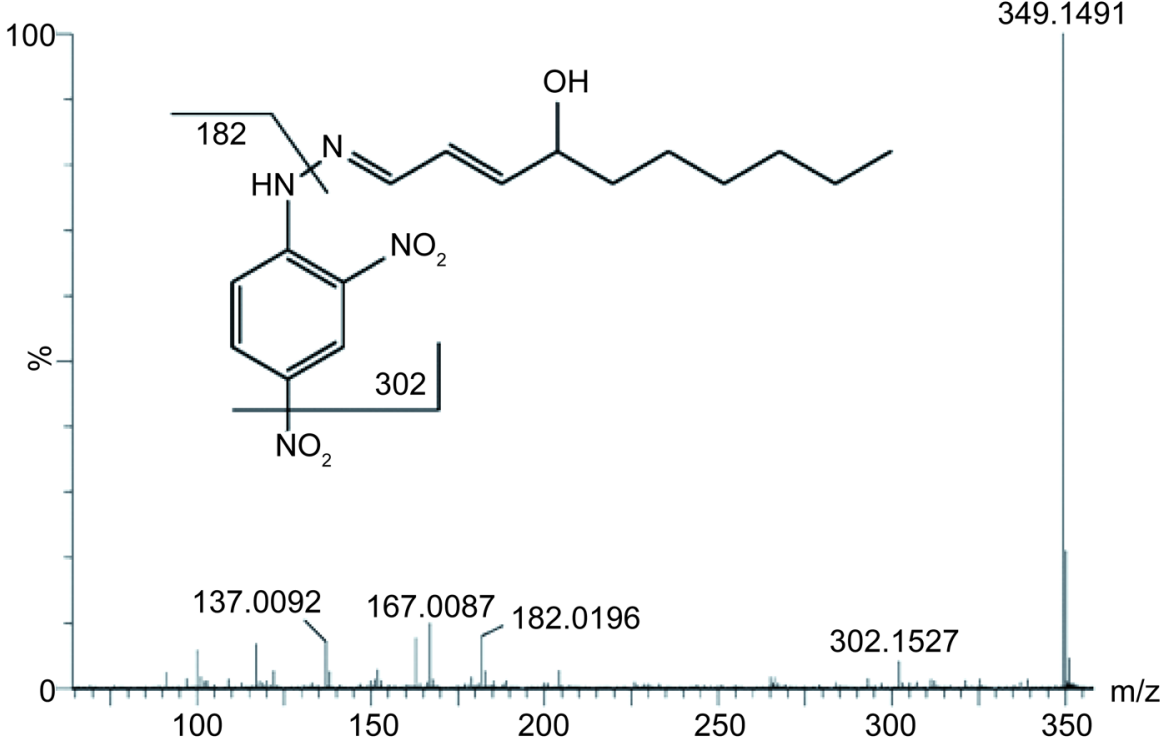

Figure 5. LC/MS trace of 4-hydroxy-2-trans-decenal (HDE)-2,4-dinitrophenylhydrazine(DNPH) standard (a), its presence in two pooled $+n-3$ urine samples (b and c), and its MS/MS fragmentogram (d), with HDE-DNPH structure).

oxidation of ALA does not produce quantifiable levels of $\mathrm{HNE}$, in contrast to linoleic acid (C18:2 n - 6) [10]. As discussed before, this is the first study to document an increase in HDE excretion from in vivo lipid peroxidation of dietary $\mathrm{n}-3$, which was confirmed by LC/MS. A further experiment is needed to identify unknown compounds $\mathrm{E}$ and $\mathrm{G}$, but it is plausible that they are longer chain polar lipophilic aldehydes produced from lipid peroxidation of $n-3$. Significant changes in total or individual non- 
polar lipophilic aldehydes were not found in the present experiment following the $n-3$ diet treatment in human subjects. Although not significant, there seemed to be increased concentrations of these compounds following the $n-3$ diet, suggesting maybe an overall increase in their production.

Through highly sensitive LC/MS analysis, we were able to confirm the significantly increased concentrations of HDE following the $+n-3$ diet. This is the first study to document urinary excretion of HDE from dietary fat intake, as well as its significantly increased concentration following a diet containing 3\% of calories from $n-3$. These results suggest that HDE is produced from in vivo lipid peroxidation of $n-3$. Although research examining $\mathrm{HDE}$ is very limited, it is plausible that since this compound shares the functional groups of HHE and HNE and differs only in its carbon chain length, it seems probable that it also binds proteins and DNA, potentially resulting in cellular mutations and death.

In conclusion, we demonstrated that a low-fat diet with $3 \%$ of energy from $n-3$ (primarily as ALA) significantly increased total polar lipophilic aldehydes from in vivo lipid peroxidation. Future research utilizing highly sensitive techniques to characterize urinary lipid peroxidation metabolites from diets with a range of $\mathrm{n}-3$ concentrations will help establish at what level $n-3$ are no longer beneficial and may become detrimental to human health. It would also be interesting to find out how dietary $\alpha$-tocopherol administration alleviates the formation of HHE from $n-3$.

\section{Acknowledgements}

Funding for this work was provided by grants from the Department of Defense (W81XWH-04-1-0448 and W81XWH-06-1-0778), the National Center for Research Resources at the National Institutes of Health (MO1-RR00400), and the USDA Agricultural Experiment Station. CAW and LRY were supported by Award Number T32CA132670 from the National Cancer Institute. The content is solely the responsibility of the authors and does not necessarily represent the official views of the National Cancer Institute or the National Institutes of Health. Salmon of the Americas, Inc., donated salmon fillets for the controlled diets.

\section{Conflicts of Interest}

The authors declare no conflicts of interest.

\section{References}

[1] Seppanen, C.M. and Csallany, A.S. (2002) Formation of 4-Hydroxynonenal, a Toxic Aldehyde, in Soybean Oil at Frying Temperature. Journal of the American Oil Chemists Society, 79, 1033-1038. http://dx.doi.org/10.1007/s11746-002-0598-z

[2] Esterbauer, H., Schaur, R.J. and Zollner, H. (1991) Chemistry and Biochemistry of 4-Hydroxynonenal, Malonaldehyde, and Related Aldehydes. Free Radical Biology and Medicine, 11, 81-128. http://dx.doi.org/10.1016/0891-5849(91)90192-6

[3] Uchida, K. (2003) 4-Hydroxy-2-Nonenal: A Product and Mediator of Oxidative Stress. Progress in Lipid Research, 42, 318-343. http://dx.doi.org/10.1016/S0163-7827(03)00014-6 
[4] Negre-Salvayre, A., Coatrieux, C., Ingeueneau, C. and Salvayre, R. (2008) Advanced Lipid Peroxidation End Products in Oxidative Damage to Proteins. Potential Role in Diseases and Therapeutic Prospects for the Inhibitors. British Journal of Pharmacology, 153, 6-20. http://dx.doi.org/10.1038/sj.bjp.0707395

[5] Kim, S.-S., Gallaher, D.D. and Csallany, A.S. (2000) Vitamin E and Probucol Reduce Urinary Lipophilic Aldehydes and Renal Enlargement in Streptozotocin-Induced Diabetic Rats. Lipids, 35, 1225-1237. http://dx.doi.org/10.1007/s11745-000-0639-2

[6] Begin, M.E., Ells, G. and Horrobin, D.F. (1988) Polyunsaturated Fatty Acid-Induced Cytotoxicity against Tumor Cells and Its Relationship to Lipid Peroxidation. Journal of the National Cancer Institute, 80, 188-194. http://dx.doi.org/10.1093/jnci/80.3.188

[7] Salo, D.C., Lin, S.W., Pacifici, R.E. and Davies, K.J. (1988) Superoxide Dismutase Is Preferentially Degraded by a Proteolytic System form Red Blood Cells Following Oxidative Modification by Hydrogen Peroxide. Free Radical Biology \& Medicine, 5, 335-339. http://dx.doi.org/10.1016/0891-5849(88)90105-0

[8] Gueraud, F., Tache, S., Steghens, J.-P., Milkovic, L., Borovic-Sunjic, S., Zarkovic, N., Gaultier, E., Naud, N., Hilies-Toussaint, C., Pierre, F. and Priymenko, N. (2015) Dietary Polyunsaturated Fatty Acids and Heme Iron Induce Oxidative Stress Biomarkers and a Cancer Promoting Environment in the Colon of Rats. Free Radical Biology \& Medicine, 83, 192 200. http://dx.doi.org/10.1016/j.freeradbiomed.2015.02.023

[9] Keller, J., Baradat, M., Jouanin, I., Debrauwer, L. and Gueraud, F. (2015) “Twin Peaks": Searching for 4-Hydroxynonenal Urinary Metaboites after Oral Administration in Rats. Redox Biology, 4, 136-148. http://dx.doi.org/10.1016/j.redox.2014.12.016

[10] Han, I.H. and Csallany, A.S. (2009) Formation of Toxic Alpha, Beta-Unsaturated 4-Hydroxy-Aldehydes in Thermally Oxidized Fatty Acid Methyl Esters. Journal of the American Oil Chemists' Society, 86, 253-260. http://dx.doi.org/10.1007/s11746-008-1343-6

[11] Calzada, C., Colas, R., Guillot, N., Guichardant, M., Laville, M., Vericel, E. and Lagarde, M. (2010) Subgram Daily Supplementation with Docosahexaenoic Acid Protects Low-Density Lipoproteins from Oxidation in Healthy Men. Atherosclerosis, 208, 467-472. http://dx.doi.org/10.1016/j.atherosclerosis.2009.07.049

[12] Young, L.R., Kurzer, M.S., Thomas, W., Redmon, J.B. and Raatz, S.K. (2011) Effect of Diet and Omega-3 Fatty Acids on Urinary Eicosanoids and Sex Hormone Concentrations in Postmenopausal Women: A Randomized Controlled Feeding Trial. Nutrition and Cancer, 63, 930-939. http://dx.doi.org/10.1080/01635581.2011.589957

[13] Esterbauer, H., Cheeseman, K.H., Dianzani, M.U., Poli, G. and Slater, T.F. (1982) Separation and Characterization of the Aldehydic Products of Lipid Peroxidation Stimulated by ADP-Fe ${ }^{2+}$ in rat Liver Microsomes. Biochemistry Journal, 208, 129-140. http://dx.doi.org/10.1042/bj2080129

[14] Kim, S.-S., Gallaher, D.D. and Csallany, A.S. (1999) Lipophilic Aldehydes and Related Carbonyl Compounds in Rat and Human Urine. Lipids, 34, 489-496. http://dx.doi.org/10.1007/s11745-999-0389-1

[15] Csallany, A.S., Kim, S.-S. and Gallaher, D.D. (2000) Response of Urinary Lipophilic Aldehydes and Related Carbonyl Compounds to Factors that Stimulate Lipid Peroxidation in Vivo. Lipids, 35, 855-862. http://dx.doi.org/10.1007/S11745-000-0594-y

[16] Fritz, K.L., Seppanen, C., Kurzer, M.S. and Csallany, A.S. (2003) The in Vivo Antioxidant Activity of Soybean Isoflavones in Human Subjects. Nutrition Research, 23, 479-487. http://dx.doi.org/10.1016/S0271-5317(03)00005-8

[17] Yao, D., Shi, X., Wang, L., Gosnell, B.A. and Chen, C. (2013) Characterization of Differen- 
tial Cocaine Metabolism in Mouse and Rat through Metabolomics-Guided Metabolite Profiling. Drug Metabolism and Disposition, 41, 79-88.

http://dx.doi.org/10.1124/dmd.112.048678

[18] Turley, E., Wallace, J., Gilmore, W. and Strain, J. (1998) Fish Oil Supplementation with and without Added Vitamin E Differentially Modulates Plasma Antioxidant Concentrations in Healthy Women. Lipids, 33, 1163-1167. http://dx.doi.org/10.1007/s11745-998-0319-2

\section{Abbreviations}

ALA: $\alpha$-linolenic acid;

DHA: docosahexaenoic acid;

DNPH: 2,4-dinitrophenylhydrazine;

EPA: eicosapentaenoic acid;

HDE: 4-hydroxy-2-trans-decenal;

HHE: 4-hydroxy-2-trans-hexenal;

HNE: 4-hydroxy-2-trans-nonenal;

HOE: 4-hydroxy-2-trans-octenal;

HPLC: high-performance liquid chromatography;

LC/MS: liquid chromatography-mass spectrometry;

n - 3: omega-3 fatty acids;

LDL: low-density lipoprotein;

MUFA: monounsaturated fatty acids;

PUFA: polyunsaturated fatty acids;

SFA: saturated fatty acids;

TLC: thin layer chromatography.

Submit or recommend next manuscript to SCIRP and we will provide best service for you:

Accepting pre-submission inquiries through Email, Facebook, LinkedIn, Twitter, etc.

A wide selection of journals (inclusive of 9 subjects, more than 200 journals)

Providing 24-hour high-quality service

User-friendly online submission system

Fair and swift peer-review system

Efficient typesetting and proofreading procedure

Display of the result of downloads and visits, as well as the number of cited articles

Maximum dissemination of your research work

Submit your manuscript at: http://papersubmission.scirp.org/

Or contact fns@scirp.org 